\title{
Traditional feeding, nutritional transition and food security
}

\section{Opinion}

The diet of the population is today a real problem, not only of public health, but of society, both in the industrialized countries as in those in the course of development. Over the past decades, the issue of malnutrition in Developing Countries (LDCs) has mainly been addressed in terms of undernourishment and its corollary of deficiencies: protein-caloric, mineral and vitamin deficiencies. More recently, it has emerged that our societies often face a double nutritional burden, in this case, undernourishment often linked to under nutrition and over-nutrition resulting from overeating with its roster of health consequences.

To meet the challenge of malnutrition, two main types of solutions are adopted; the supply of food stocks for food-deficient populations and the emergence of standard or even universal diets to ensure better health for populations. However, between these two major ones, which are food inaccessibility and food over-consumption, there are some finer causes, linked to our habits or behaviors and our relationship to food:

The massive influx of food from global solidarity makes it possible, of course, to solve emergencies and save many lives. However, this has the effect of modifying the diet of the populations concerned and often creating a food dependency that puts Amal in the position of food sovereignty. In some cases, this situation is more than worrying. To prevent the pandemic of global obesity, especially in the Western world, solutions are quickly oriented towards the adoption of miracle regimes that are supposed to solve all the evils of malnutrition, as if by magic wand. The Cretan, Mediterranean or Asian diets (Okinawa type) are thus cited as examples, when they are not presented as perfect examples of a capable diet.

To ensure respectable health and longevity (many books are devoted to it). Too often, the socio-environmental aspects are ignored in favor of central Laplace made to food, and to the detriment of other aspects of an art of living in which the food model participates. In order to fully understand the role of diets and their impact on our health, and even our longevity, we need to look at traditional ways of feeding populations.

\section{Traditional feeding}

The traditional diet for each society is the result of a number of experiences accumulated over several generations, through adoptions and rejections of food and new consumption patterns. But also, through periods of famine and overabundance, epidemics of wars and critical changes in the environment.

This diet, based on major foods that is frequently and readily available, must be able to cover essential energy, hedonic and social needs.

Traditional food takes into account not only the selection and choice of food, but also consumption patterns. Indeed, these modes integrate rites and rituals specific to each population. They participate in its cultural and even cultural profile in some cases. Aids, advice or recommendations on population nutrition must therefore take
Volume 4 Issue 2 - 2017

\author{
Séraphin Kati Coulibaly \\ Department of Laboratory of Nutrition and Pharmacology UFR \\ Biosciences, University Felix Houphouet-Boigny, Africa
}

Correspondence: Séraphin Kati Coulibaly, Department of Laboratory of Nutrition and Pharmacology UFR Biosciences, University Felix Houphouet-Boigny, Africa,

Email katicoulibalys@yahoo.fr

Received: March 07, 2017| Published: March 14, 2017

this dimension into account, even if the global cliché shows that most populations are trained in the great movement of a nutritional transition.

\section{Nutritional transition}

In many African countries, populations, especially in urban areas, are faced with a sometimes profound change in their eating habits.

This results in a gradual or abrupt transition from a conventional type of supply to an industrial type feed. The latter type is based on the often excessive use of refined products, large providers of carbohydrates and lipids in the form of empty calories. For some populations, nutritional transition is desired and chosen. But for others, it is suffered and in fact entails serious consequences on the medium and long term.

\section{Dietetic conscience}

Governments have taken stock of the nutritional dangers that threaten global public health. The temptation of medicalization of our diet is great. There is, however, a saving alternative through the adoption of a new dietary code. To this end, awareness at the highest level, notably the United Nations, which is at the origin of the Scaling up Nutrition (SUN) initiative, is a real boon for the global nutritional situation. Indeed, the effort to coordinate the problems of food at the level of the planet is no longer oriented towards a standardization of dietary habits, but towards the harmonization of strategies that ensure local populations, food security and health Adequate for their harmonious development.

The adoption by various states of national plans to reduce, under nutrition, and over-nutrition, together with their associated tares, is essential in helping populations to assume a Dietary Consciousness capable, whatever the hazards, of ensuring their health and a respectable longevity at the heart of environmentally friendly development.

\section{Traditional food, nutritional transitions and food safety}

The feeding of the populations is a real problem, not only for public health, but society concern, as well in the industrialized than 
in developing countries. During the previous decades, the question of the malnutrition in developing countries was essentially approached under the angle of the sub-nutrition and its corollaries of deficiencies; protein-calorie, mineral and vitamin. More recently, it seemed that our societies often faced a double nutritional burden, in this particular case; the sub-nutrition is bound to malnutrition and a surnutrition ensuing from an overeating with its rosary of consequences

The massive contribution of food stemming from the world solidarity allows, certainly to solve emergency situations and to save so numerous lives. However, it consequently modifies the diet of the concerned populations and creates often a food dependence which puts for the worse the food sovereignty of these populations. In certain cases this situation is more than worrisome.

To prevent the pandemic aspect of the world obesity, especially in developed countries, the solutions are fast directed to the adoption of miracle, sensible diets to solve, as by a wave of the wand, all the troubles of the malnutrition. The Cretan, Mediterranean or Asian regimen like Okinawa diet, are so quoted as a model, when they are not presented as perfect examples

\section{Traditional food}

The traditional food for every community is the result of a sum of experiences accumulated on several generations, through, the adoptions and the rejections of food and new modes of consumption. But, also through the periods of starvation and profusion, war epidemics and critical changes of the environment. This food based on major food frequently and easily available must be capable of covering the essential needs so energy, hedonic, that social.

The traditional food takes into account not only the selection and the choice of food, but especially the modes of consumption which integrate rites and ritual appropriate to every population and participate in its cultural profile

\section{Nutritional transitions}

In numerous African countries the populations, especially in urban zones face a modification, sometimes deep, of their food habits. It is characterized by a progressive or abrupt passage from a traditional food type to industrial food type based on the use often in excess, of refined products big suppliers of carbohydrates and lipids in the form of empty calories.

For certain populations the nutritional transition is wanted and chosen, but for others, it is undergone and pulled de facto, with high consequences on the mean and the long term.

\section{Dietary Consciousness}

Public authorities took the measure of the nutritional dangers of order which threaten the world public health. The temptation of a medicalization of our feeding is big, there is however a saving alternative through the adoption of a new dietary code.

For that purpose, the awareness at the level of the high authorities of the United Nations which is at the origin of the initiative SUN (Scaling up Nutrition) is a real chance of a lifetime for the world nutritional situation. Indeed, the effort of coordination of the problems of the food at the level of the planet is not directed to a standardization of the food modes, but to a harmonization of the strategies which assure the local populations, the food safety and the adequate health.

\section{Acknowledgements}

None.

The author declares no conflict of interest. 